\title{
LASER SHOCK COMPACTION OF POROUS MATERIALS
}

\author{
D. ZAGOURI, J.-P. ROMAIN* , B. DUBRUJEAUD ${ }^{* *}$ and M. JEANDIN**
}

Peugeot S.A., Etudes et Recherches, D.R.A.S., Route de Gizy, F-78140 Vélizy, France

"Laboratoire d'Energétique et de Détonique, URA-CNRS 193, ENSMA, F-86034 Poitiers, France

${ }^{*}$ Centre des Matériaux Pierre-Marie Fourt, Ecole des Mines de Paris, BP. 87, F-91003 Evry, France

\begin{abstract}
Laser-driven shock-waves are used as a surface treatement for compacting porous materials. The compaction depth is typically a few hundreds of microns. The behavior of the porous medium is described through a compaction model based on the $P$ - $\alpha$ theory of Herrmann. This model has been introduced into a one-dimensional finite difference hydrodynamic code describing the behavior of a target under the action of a laser-generated shock-wave. The code enables us to compute the compaction depth as a function of irradiation conditions, nature and initial porosity of the material. Experiments are performed an aluminum powder. Samples are observed by optical microscopy. The residual porosity is estimated by image analysis. Experimental results and computed compaction profiles correlate well.
\end{abstract}

\section{1.- Introduction}

Dynamic consolidation of porous materials is usually performed with classical shock generators (explosives or flyers impact) providing uniformly compacted parts. Recently, the possibility of compacting powders with laser-driven shock waves was demonstrated $/ 1 /$. In this case, the pressure is applied on the target during a very short time (1-100 ns) leading to a densification on a small depth (a few hundreds of microns).

The purpose of this paper is to show experimental results obtained by laser shock processing and to compare these results with computer simulations.

\section{2.- Porous model}

The behavior of a porous material under shock loading is described by a widely used model (P- $\alpha$ model) proposed by W. Herrmann $/ 2$ / and modified by M. M. Carroll and A. C. Holt $/ 3 /$. The pressure $\mathrm{P}$ is given as a function of the specific volume $\mathrm{V}$ and the specific internal energy $\mathrm{E}$ of the porous material by the relation :

$$
\mathrm{P}=\frac{\mathrm{f}\left(\frac{\mathrm{V}}{\alpha}, \mathrm{E}\right)}{\alpha}
$$

with $\alpha=\frac{\mathrm{V}}{\mathrm{Vs}}$

(Vs specific volume of the matrix material).

The function $f$ is assumed to be the same as the function that relates the pressure Ps to the specific volume Vs and the specific internal energy Es for the solid material (Ps $=f(V s$, Es )). 
The variations of the porosity $\alpha$ are expressed by a function of the specific volume $V$ proposed by $D$. E. Maxwell /4/ $/ 5 /$ depending on a parameter $\beta$.

The model includes an initial elastic compression up to a pressure Pe.

Resulting Hugoniot curves are shown on figure 1 as a function of the parameter $\beta$.

This model has been introduced into a 1-D finite difference hydrodynamic code.

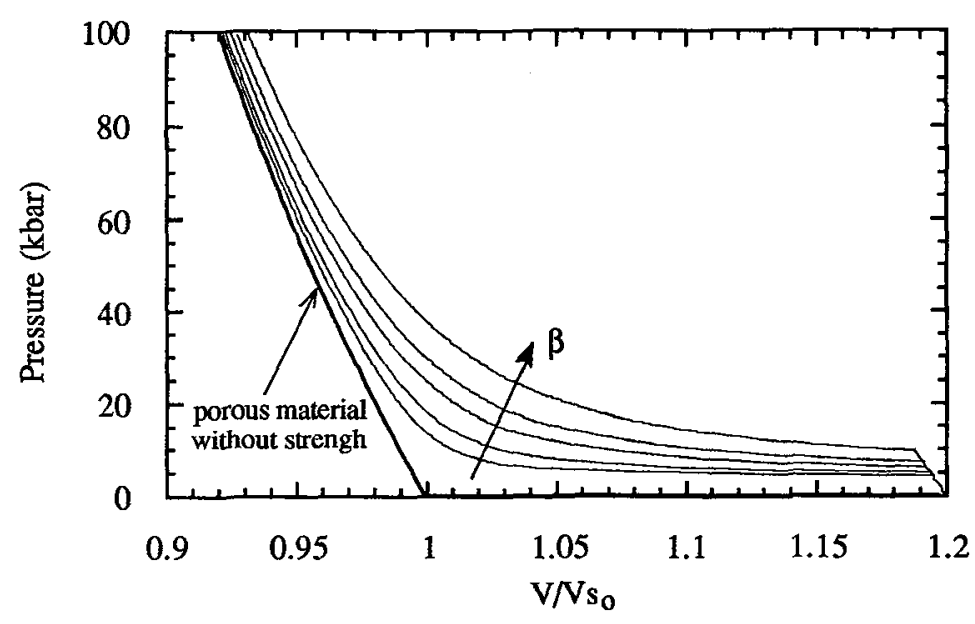

Figure 1

Hugoniot curves for porous aluminum $\left(\alpha_{0}=1.2\right)$.

Influence of the parameter $\beta$.

\section{3.- Experiment and analysis of compaction of an aluminum powder}

The set-up is shown on figure 2. The samples are covered with a transparent glass window acting as a confinement for the plasma generated by absorption of the incident laser radiation on the target surface. This process increases the applied pressure and its duration in comparison with a bare target irradiation. An absorbing layer which can be a metal foil or a layer of black paint protects the sample from melting.

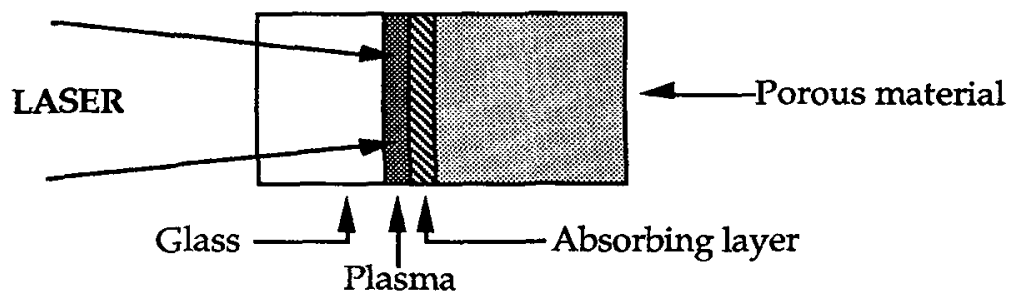

Figure 2

Experimental set-up. 
Experiments were performed on aluminum powders at $80 \%$ crystal density $\left(\alpha_{0}=1.25\right)$.

The figure $3 \mathrm{a}$ shows the cross section of a target which was submitted to the following irradiation conditions :

$$
\begin{aligned}
& \text { - wavelength }: \lambda=1.06 \mu \mathrm{m} \\
& \text { - energy }: \mathrm{E}=21.5 \mathrm{~J} \\
& \text { - pulse duration }: \tau=8.8 \mathrm{~ns} \\
& \text { - incident intensity: } \mathrm{I}=8.6 \mathrm{GW} / \mathrm{cm}^{2}
\end{aligned}
$$

With a coupling efficiency of $16 \%$, the applied pressure is estimated to be about $60 \mathrm{kbar} / 6 / \pi /$.

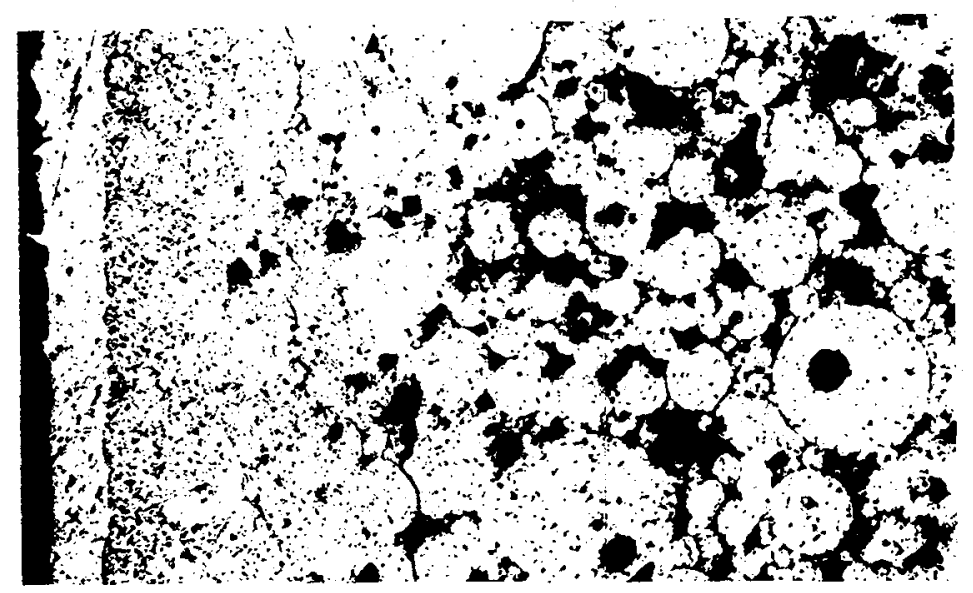

$-\mathbf{a}-$

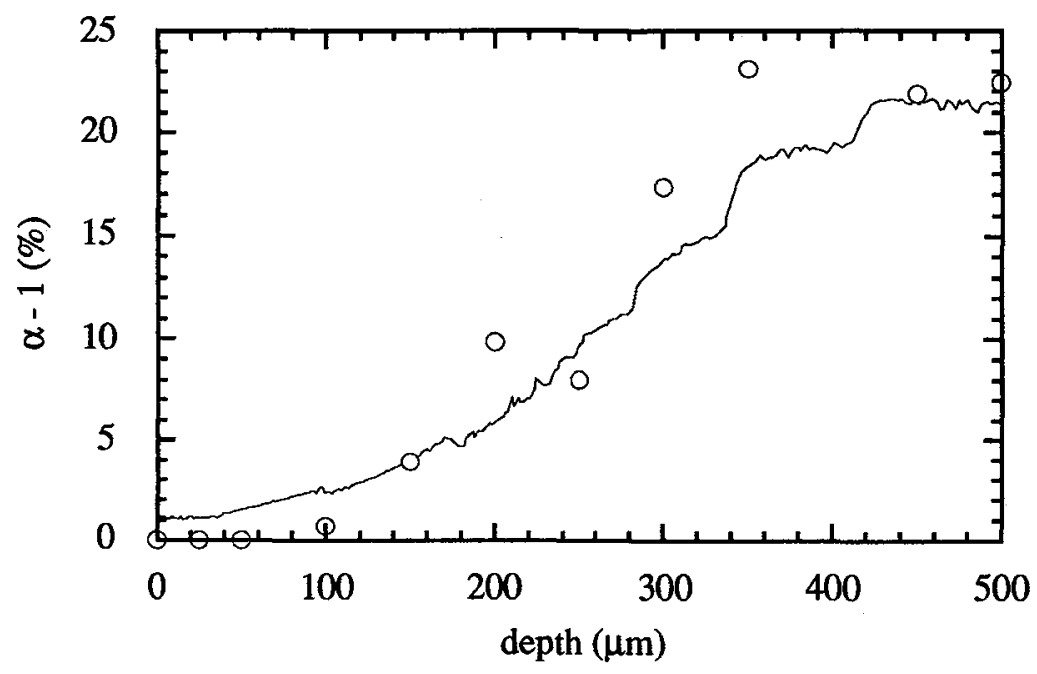

$-b-$

Figure 3

Cross section of the irradiated aluminum sample (a).

Computed profile and experimental points (b). 
The residual porosity is calculated by image analysis and the experimental results are in good agreement with the computed profil (figure $3 b$ )

\section{4.- Conclusion}

Laser-driven shock waves are able to achieve surface densification of porous materials. Experiments show that the compaction depth is about a few hundreds of microns, depending on the irradiation conditions and on the nature and the initial porosity of the material.

Computer simulations can be used to predict the compaction depth as a function of the irradiation conditions for a given initial porosity of the material.

\section{References}

/1/ J.-P. ROMAIN \& D. ZAGOURI, in Shock Waves and High Strain Rate Phenomena, eds. M. A. Meyers, L. E. Murr and K. P. Staudhammer, M. Dekker New York (1991)

/2/ W. HERRMANN, J. Appl. Phys. 40, 3490 (1969)

3/ M. M. CARROLL \& A. C. HOLT, J. Appl. Phys. 43, 759 (1972)

14/ D. E. MAXWELL, Science Applications, San Leandro, SATR 82-4 (1982)

/5/ J. E. REAUGH, J. Appl. Phys.61, 962 (1987)

/6/ P. DARQUEY, J.-P. ROMAIN, J. Appl. Phys. 68, 1926 (1990)

17/ J. FOURNIER, R. FABBRO, P. BALLARD, J. VIRMONT, Proceedings, $19^{\text {th }}$ ECLIM, Madrid (1988) 